\title{
Observations and modelling of earth's transmission spectrum through lunar eclipses: A window to transiting exoplanet characterization
}

\author{
E. Pallé ${ }^{1}$, Antonio García Muñoz ${ }^{1}$, Maria R. Zapatero Osorio ${ }^{2}$, \\ Pilar Montañés-Rodríguez ${ }^{1}$, Rafael Barrena $^{1}$ \\ and Eduardo L. Martín ${ }^{2}$ \\ ${ }^{1}$ Instituto de Astrofisica de Canarias, La Laguna, Tenerife, Spain \\ email: epalle@iac.es \\ ${ }^{2}$ Centro de Astrobiología, CSIC-INTA, Madrid, Spain
}

\begin{abstract}
Recently we were able to retrieve the Earth's transmission spectrum through lunar eclipse observations. This spectrum showed that the depth of most molecular species was stronger than models had anticipated. The presence of other atmospheric signatures, such as atmospheric dimers, were also present in the spectrum. We have been developing a radiative transfer code able to reproduce the Earth's transmission spectra at different depths into the penumbra and umbra, and taking into account transmission, refraction, and multiple scattering. Here we discuss the results to date and the work ahead.
\end{abstract}

Keywords. astrobiology, radiative transfer, radiation mechanisms: general, planets and satellites: general, Earth

\section{Introduction}

The observational technique of transmission spectroscopy during extrasolar planet transits allow us to obtain the transmission spectrum of a planet atmosphere. When the transit occurs part of the light that reaches the observer has crossed the planetary atmosphere and contains the signatures of its spectroscopically-active component gaseous species. Based on HST high-precision spectrophotometric observations, Charbonneau et al. (2002) detected the absorption from sodium in the atmosphere of HD209458b, while Richardson et al. (2007) reported its infrared spectra (7.5-13.2 $\mu \mathrm{m})$. Using the Spitzer telescope and this same methodology, Tinetti et al. (2007) and Swain et al. (2008) have recently reported the presence of water and methane, respectively, in the atmosphere of planet HD189733b. Furthermore, the large number of transiting planets expected to be discovered by CoRot and KEPLER and the advent of more sensitive instruments like the JWST, makes this technique extremely promising for the characterization of exoplanetary atmospheres. But what about the detection and characterization of small, rocky planets? To be ready to complete such identifications in the case of terrestrial planets, one requires globally-integrated observations of the Earth and the rest of rocky planets of the solar system. Specially interesting are the observations of the transmitted spectra of their atmospheres. The characterization of the spectral features in our planet's transmission spectra, as if it were observed from a distant star, can be achieved through the earthshine observations during a lunar eclipse, and constitute an important benchmark for future exoplanets studies. 


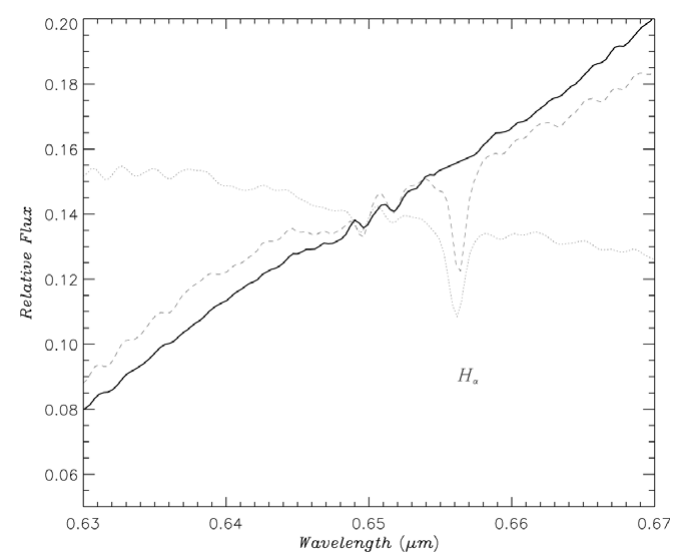

Figure 1. A detail of the umbra spectrum (broke line), the bright Moon spectrum (dotted line) and their ratio spectrum (umbra/bright) around the hydrogen alpha $\left(H_{\alpha}\right)$ line $(0.6568 \mu \mathrm{m})$. The $H_{\alpha}$ solar line is a greatw example to illustrate the high $\mathrm{S} / \mathrm{N}$ of our observations. In this figure, one can see that the $H_{\alpha}$ is present in the raw spectra of the umbra and the bright Moon, but not in the final transmission spectra.

\section{Eclipse observations and modeling}

A lunar eclipse is a relatively rare event that occurs when the Moon enters the cone of shadow cast by the Sun-lit Earth. From within the umbra of the eclipse, the planet prevents a direct view of the solar disk. The Moon in umbra, however, is not entirely dark because a fraction of the solar photons that penetrate the terrestrial atmosphere are either refracted or scattered towards the Moon. This fraction, when normalized by the solar irradiance, is a measure of the transmissivity of the Earth's atmosphere, while the spectral features of the Sun, the Moon's albedo and the local telluric atmosphere are canceled (see Figure 1). The fraction is strongly dependent on both the composition of the extended region in the Earth's terminator traversed by the photons and on the photons' wavelength. In the umbra, the pathlength traversed by photons is long, so that weakly absorbing gases yield prominent absorption signatures. In 2009 the first spectrum of a lunar eclipse, with data taken at two ground-based telescopes in La Palma (Spain), was published by Pallé et al. (2009). Our 0.36-2.4 $\mu \mathrm{m}$ spectrum of the partial eclipse of 16 August 2008 shows clear evidence of a number of biologically relevant molecules such as $\mathrm{O}_{3}, \mathrm{O}_{2}, \mathrm{H}_{2} \mathrm{O}, \mathrm{CH}_{4}$ and $\mathrm{CO}_{2}$, and the dimers $\mathrm{O}_{2}-\mathrm{O}_{2}$ and $\mathrm{O}_{2}-\mathrm{N}_{2}$ (see Figure 2).

Simulations using the empirical Earth's transmission spectrum, and the stellar spectra for a variety of stellar types, indicate that with the new generation of extremely large telescopes, such as the proposed 42-meter European Extremely Large Telescope(E-ELT), we could be capable of retrieving the transmission spectrum of an Earth-like planet around very cool stars and brown dwarfs $\left(T_{\text {eff }} \leqslant \sim 3100 \mathrm{~K}\right)$ (Palle et al. 2011$)$.

The classical theory of lunar eclipses, nicely summarized by Link (1962), is built on the premise that the sunlight scattered by the gases and particles in the atmosphere contributes negligibly to the brightness of the eclipsed Moon. Our recent modeling effort (García Muñoz and Pallé, 2011) revisits the lunar eclipse theory, extending it to accommodate spectrally-resolved observations and addressing the role of scattered sunlight. Predictions of both direct and diffuse sunlight are produced by integrating the radiative transfer equations over the Earth's disk. We find that omitting scattered sunlight is an acceptable approximation for low and moderate aerosol loadings at visible and longer wavelengths. However, towards the ultraviolet, or at times when the atmosphere contains elevated aerosol amounts, the relative significance of direct and diffuse sunlight may 

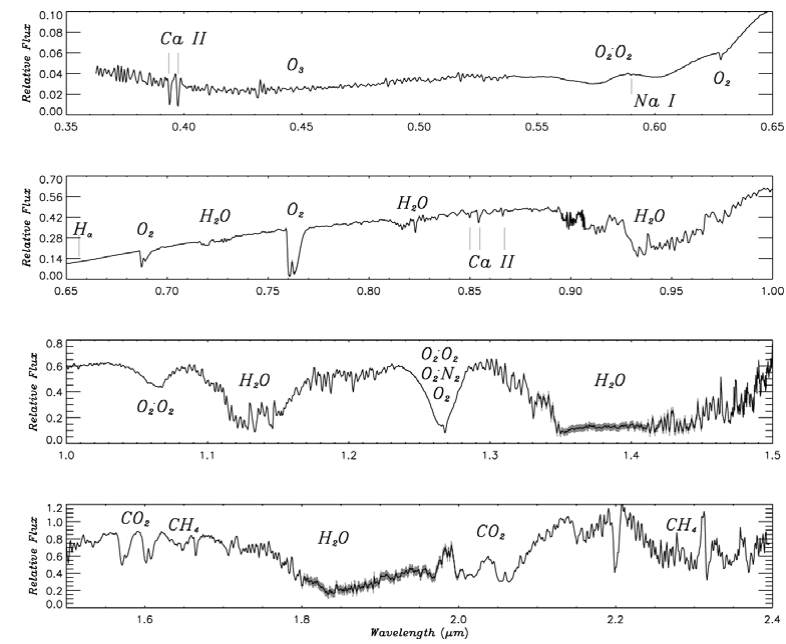

Figure 2. The Earth's transmission spectrum from 0.36 to $2.40 \mu \mathrm{m}$. The major atmospheric features of the spectrum are marked. Adapted from Palle et al. (2009).

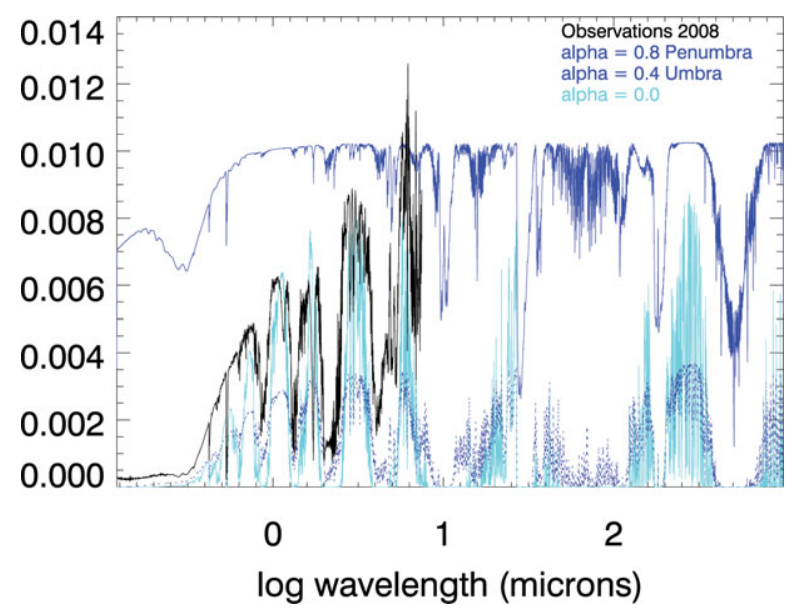

Figure 3. Modeled Moon-reflected radiances for $\Delta \lambda / \lambda 1000$ at various $\alpha$ angles during the eclipse covering the spectral range from 0.4 to 25 micron. Y scale is in arbitrary units.

reverse (García Muñoz et al., 2011). The conclusion is supported by two distinct features, namely the spectrums tail at short wavelengths and the unequal absorption by the $\left(\mathrm{O}_{2}\right)_{2}$ dimers at two nearby bands. Our findings are consistent with the elevated aerosol loadings reported for several months at high northern latitudes following the 78 August 2008 Kasatochi volcano eruption in Alaska. We also find that lunar eclipse spectra will reveal the presence on enhanced background aerosol levels up to 4 or 5 years after a major volcanic eruption.

\section{Future work}

As shown in Figure 1, model calculations predict that the structure of the spectrum of Moon-reflected light depends strongly on the phase of the eclipse, with both the total amount of light and its spectral distribution varying (see Figure 3). To quantify this we define a parameter, alpha, which is defined as the angle between the center of the umbral 
disk and the region of the Moon that we are sampling at each time. This dynamical behavior involves an additional difficulty in the characterization of the Moon-reflected light. Such observations can be gathered with 8 to 10 meter class telescopes at very high SNRs, and the complete temporal evolution of the atmospheric signatures can be followed over the duration of the lunar eclipse.

We intend to conduct eclipse observations at high spectral resolution and high temporal cadence to help confirm the model calculations and to improve the theory of lunar eclipses as it stands at present. This is necessary for optimizing strategies to search for Earth analogs. The observations will also serve as input data for new generation planet-hunting high-resolution spectrographs.

\section{References}

Charbonneau, D. et al. 2002, ApJ, 568, 377

García-Muñoz, A. \& Pallé, E. 2011, JQSRT, in press, DOI: 10.1016/j.jqsrt.2011.03.017

García-Muñoz, A., et al. 2011, GRL, 38, L14805

Link, F. 1962, Physics and Astronomy of the Moon, 560, 161

Pallé, E., et al. 2009, Nature, 459, 814

Pallé, E., Zapatero-Osorio, M. R., \& Garcia-Munoz, A. 2011, ApJ, 728, 19

Richardson, L. J., et al. 2007, Nature, 445, 892

Swain, M. R., Vasisht, G., \& Tinetti, G. 2008, Nature, 452, 329

Tinetti, G., et al. 2007, Nature, 448, 169 